\title{
Neurocognitive effects of HCV, methamphetamine abuse, and HIV: multiple risks and mechanisms Scott L Letendre
}

\author{
Address: University of California, San Diego, California, USA \\ from International Society on Brain and Behaviour: 3rd International Congress on Brain and Behaviour \\ Thessaloniki, Greece. 28 November - 2 December 2007 \\ Published: 17 April 2008 \\ Annals of General Psychiatry 2008, 7(Suppl I):S39 doi:I0.I I86/I744-859X-7-SI-S39
}

This abstract is available from: http://www.annals-general-psychiatry.com/content/7/SI/S39

(C) 2008 Letendre; licensee BioMed Central Ltd.

HIV-infected individuals are at risk for a spectrum of neurocognitive disorders. Research performed in developed and resource-limited settings indicates that over half of HIV-infected individuals may have neurocognitive impairment of at least mild severity. An important issue complicating the pathogenesis of these disorders is the coexistence of multiple neurotoxic conditions. Two important comorbidities are co-infection with Hepatitis C Virus (HCV) and use of stimulants, such as methamphetamine (MA).

The WHO estimates that $3 \%$ of the world's population has been infected with HCV, leaving 170 million chronically infected. HCV co-infects approximately a third of all HIVinfected individuals, $75-90 \%$ of HIV-infected intravenous drug users, and nearly $100 \%$ of some groups of HIVinfected hemophiliacs. The longer life expectancy of HIVinfected individuals since the advent of combination antiretroviral therapy has highlighted the impact of chronic HCV, which has emerged as a frequent cause of death in HIV-infected people.

A growing body of literature fuels the notion that $\mathrm{HCV}$ can injure the brain. First, HCV can infect resident (e.g., astrocytes) and migrating (e.g., macrophages) cells of the central nervous system (CNS). Second, HCV can adapt to neural cells, as evidenced by phylogenetic compartmentalization. Third, the neuropsychological (NP) performance of HCV-infected individuals is more likely to be impaired than that of uninfected individuals. Fourth, magnetic resonance spectroscopy demonstrates that HCVinfected individuals have elevated choline-to-creatine ratios in the basal ganglia and white matter, suggesting inflammation, and reduced $\mathrm{N}$-acetyl aspartate in white matter, suggesting neuronal loss.
Chronic or heavy exposure to MA has also been associated with brain injury. MA may injure the brain by multiple mechanisms including interfering with dopamine metabolism, altering glutamate processing by astrocytes, increasing TNF-alpha expression, and worsening oxidative stress. In HIV-infected individuals, MA may also increase HIV replication.

These and other data support that each of these conditions can lead to brain injury and that their effects may be additive. The high prevalences of these conditions also highlight important public health concerns. For example, the substantial cognitive impairment in these populations may negatively affect their ability to manage complex medical therapy. As a result, their likelihood of recovery and survival - may be greatly reduced. 